
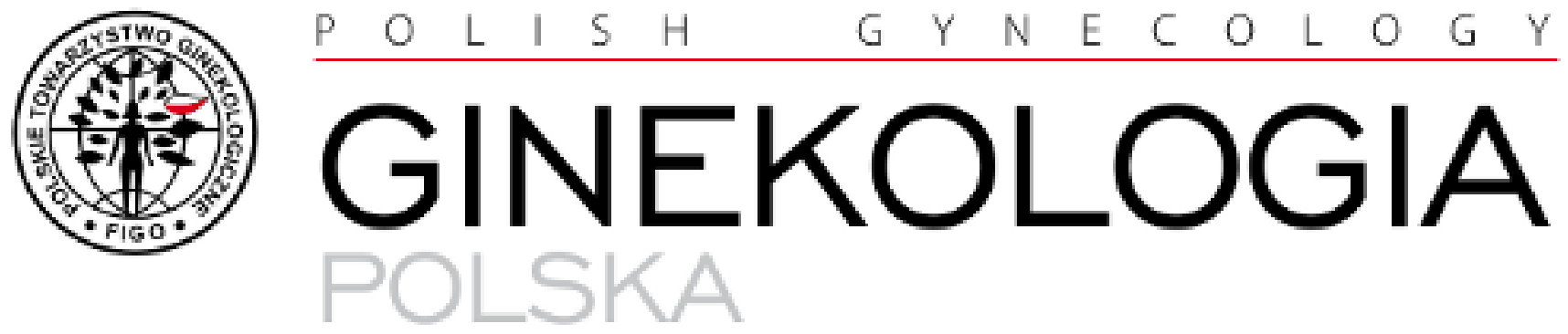

ORGAN POLSKIEGO TOWAAZYSTWA GINE KOLOGICZNEGO

THE OFFICIAL JOURNAL OF THE POLISH GYNECOLOGICAL SOCIETY

ISSN: 0017-0011

e-ISSN: $2543-6767$

\title{
Endometriosis is associated with an increased whole-blood thrombogenicity detected by a novel automated microchip flow-chamber system (T-TAS $\left.{ }^{\circledR}\right)$
}

Authors: Malgorzata Kedzia, Maciej Osinski, Urszula Mantaj, Ewa WenderOzegowska

DOI: $10.5603 / G P . a 2021.0153$

Article type: Research paper

Submitted: $2020-06-30$

Accepted: 2021-01-11

Published online: 2021-08-06

This article has been peer reviewed and published immediately upon acceptance.

It is an open access article, which means that it can be downloaded, printed, and distributed freely, provided the work is properly cited. 
Articles in "Ginekologia Polska" are listed in PubMed. 
Endometriosis is associated with an increased whole-blood thrombogenicity detected by a novel automated microchip flow-chamber system (T-TAS ${ }^{\circledR}$ )

Malgorzata Kedzia, Maciej Osinski, Urszula Mantaj, Ewa Wender-Ozegowska

Division of Reproduction, Poznan University of Medical Sciences, Poland

Short title: T-TAS ${ }^{\circledR}$ values alteration in endometriosis

\section{Corresponding author:}

Maciej Osinski

Division of Reproduction, Poznan University of Medical Sciences, Poland e-mail: maciejosinski@me.com

\section{ABSTRACT}

Objectives: Potential thrombotic and antifibrinolytic influence of endometriosis on haemostasis has been recently reported in the literature, as well as increased cardiovascular morbidity in women suffering from the disease. We performed a pilot study to assess the influence of endometriosis on the thrombus formation process under in vitro flow conditions. Material and methods: This study compared women with confirmed endometriosis $(n=23)$ surgically and control healthy subjects $(\mathrm{n}=10)$. In both groups, the same exclusion criteria were used: a prior episode of thrombosis diagnosed as acquired or inherited thrombophilia, neoplasm, and an uncertain family history of thrombosis. We evaluated the whole blood thrombogenicity using T-TAS ${ }^{\circledR}$ at a shear rate of 240 s-1 (Total-Thrombus Analysis System, Zacros, Japan).

Results: The blood clot formation initiation time (T10) and occlusion time (OT) were significantly shortened in the endometriosis group $(\mathrm{p}<0.05)$. The area under the curve (AUC30) of blood clot time formation values (BCTF) was substantially higher in the patients suffering from a disease $(\mathrm{p}=0.03)$. An increase in AUC (TTAS) values by 100 increases the risk of developing endometriosis by 1.56-fold [adjusted OR = 1.56 ( $\mathrm{p}=0.01) ;(95 \%$ CI: 1.102.18)]. Inflammatory markers (neutrophil-to-lymphocyte ratio (NLR), and the leucocyte, neutrophil, basophil, and neutrophil concentrations) were also substantially higher in the endometriosis group ( $\mathrm{p}<0.05)$. 
Conclusions: The alteration of the T-TAS and NLR values supports the thesis of a shift of the equilibrium towards thrombosis in women who have endometriosis. This phenomenon links to a state of chronic inflammation. It is detectable using a novel system for the quantitative assessment of the platelet thrombus formation process under flow conditions in vitro.

Key words: endometriosis; thrombosis; prothrombotic state

\section{INTRODUCTION}

Endometriosis is a disease defined by the occurrence of endometrial glands and stroma outside the uterine cavity [1]. It remains a relatively prevalent gynaecological disorder. That disease affects from $6 \%$ to $10 \%$ of women of reproductive age in the United States of America [2].

Despite this, the aetiology of the disorder is still elusive [3]. A growing body of scientific evidence has recently been linking endometriosis with an immunologic dysfunction, metabolic changes, the atherogenic lipid profile, oxidative stress as well as with chronic systemic inflammation $[4,5]$. Moreover, this disease may be a significant risk factor for major chronic diseases, although the underlying mechanism is not yet fully understood [6].

In contemporary research, an association of endometriosis with many common systemic comorbidities, such as cancers, allergy, autoimmune and cardiovascular diseases have been found [6-8]. Coronary heart disease (CHD) remains an example of arterial thrombosis, and in a large prospective study, laparoscopically confirmed endometriosis was associated with an increased risk of CHD episodes. That association was more influential in the younger group of women who have endometriosis. Moreover, hysterectomy/oophorectomy procedures and hormonal therapy increase the risk of CHD; however, those can only partially explain the link of CHD with endometriosis [9-11]. After adjustments for potential confounders, the relative risk of CHD in Endometriosis is 1.62-fold increased, in comparison to non-endometriosis women [6].

Various inflammatory factors such as intracellular adhesion molecule 1 (ICAM-1), interleukin-1 and interleukin-6 (IL-1 and IL-6), tumour necrosis factor-alpha $\alpha$ (TNF- $\alpha$ ) and vascular endothelial growth factor (VEGF) and vitamin D polymorphism alteration have been described in endometriotic patients [12-16]. The systemic inflammatory response results in changes in the white blood cells (WBC) and the coexistence of relative lymphocytopenia and neutrophilia has been observed $[17,18]$. Thus, the increased ratio of neutrophil-to-lymphocyte (NRL) in endometriosis women strongly suggests the occurrence of chronic inflammation in this group of patients [19]. In endometriosis, an increased level of activated macrophages and 
increased levels of the cytokines provides a local microenvironment suitable for the initiation and growth of endometriotic lesions [20].

A growing body of research supports the thesis that inflammation and haemostasis processes interact substantially with each other [21]. The coagulation modulates the inflammation, and the inflammation activates the coagulation cascade [22, 23]. The inflammatory response enhances and promotes the initiation of coagulation, increasing the risk of organ dysfunction and microvascular thrombosis [24, 25].

In endometriosis patients, the haemostasis and coagulation assay values are altered. In 2015, Wu et al., proved that in women with endometriosis, the APTT (activated partial thromboplastin time), and TT (thrombin time) are shortened while the fibrinogen concentration is elevated. Moreover, in their blood, a higher percentage of circulating degranulated platelets was found [21]. Several studies have demonstrated that those changes are involved in the development of endometriosis. Endometriosis women appear to remain in a hypercoagulable and hypofibrinolytic state due to platelet aggregation in endometriotic lesions. It provides a potential rationale for the use of anticoagulants to treat endometriosis [26].

Platelet size is an essential marker of platelet function. Mean platelet volume (MPV) remains an indicator of platelet activation, and increased values link to platelet hyperreactivity. Thus, MPV is also an important biological variable predicting the risk of arterial micro thrombotic episodes such as vascular complications in diabetes mellitus. Liu et al., showed an association of MPV with the severity of diabetic retinopathy [27].

There are numerous methods for assessing haemostasis. However, screening coagulation assays are not sensitive enough to detect hypercoagulation adequately and thus remain unable to evaluate thrombotic risk [28]. Efforts have been made to use global, integral assays to mimic and reflect aspects of haemostasis in vitro. An example of such is the Total Thrombus-formation Analysis System (T-TAS $\left.{ }^{\circledR}\right)$. This system enables the evaluation of the in vitro thrombus formation process under flow conditions. (T-TAS $\left.{ }^{\circledR}\right)$ is an innovative microchip flow-chamber system enabling quantitative assessment of the platelet thrombus formation process. This assay represents noteworthy improvements over conventional platelet function assays. The fact that T-TAS is sensitive not only to platelet aggregation but also detects alterations occurring in the fibrinolytic system is very important considering the aim of our study [29].

Many previous studies have demonstrated the influence of endometriosis on some coagulation assays, but still, conventional coagulation assays are often not sensitive enough to 
detect hypercoagulation and are unable to evaluate the risk of developing thrombosis. To date, there has been no research study assessing the influence of endometriosis on the process of thrombus formation under flow conditions in vitro.

Our study aimed to evaluate the whole blood thrombogenicity in women who have endometriosis to assess in this pilot study the occurrence of any alteration of haemostasis in this group of women.

\section{MATERIAL AND METHODS}

\section{Subjects}

We conducted the study in the Department of Reproduction at the Poznan University of Medical Sciences from December 2017 until March 2018. The analysis included 23 patients with surgically confirmed endometriosis as well as 10 healthy women, who formed the control group.

We collected the data of patients who were referred to the gynaecological ward due to a suspicion of endometriosis. All the patients from the endometriosis group suffered from at least one of the clinical symptoms and signs of the disease such as dysmenorrhea, dysuria, dyspareunia, dyschezia, subfertility, or infertility.

We took a detailed history which included a list of varied gynaecological items from every subject in the control group. All women from this group presented regular monthly menses with standard length and denied using any medications, including oral contraceptives, in the previous 12 months.

Every woman from the control group denied the occurrence of symptoms such as ever having been diagnosed or having a suspected diagnosis of endometriosis.

Moreover, in both groups, the same additional exclusion criteria were used to avoid significant confounding factors, such as an episode of venous or arterial thrombosis in the prior history, diagnosed acquired or inherited thrombophilia, neoplasm and an uncertain family history of thrombosis, pregnancy, hypertension, smoking and the occurrence of any vascular disease.

\section{Methods}

Blood samples for all tests in both groups were taken in the follicular phase of the menstrual cycle. In patients undergoing surgical procedures, blood samples were taken a day before the scheduled surgery.

\section{Standard comparative laboratory findings}


All biochemical tests were performed in the accredited laboratory of the University hospital (Central Laboratory, The Obstetrics and Gynecology Teaching Hospital of Poznan University of Medical Sciences), which holds an ISO 9000 certificate of quality management. We collected blood samples for standard conventional laboratory tests at the same time in a fasting state from the antecubital veins.

After being drawn, the blood was collected in tubes containing ethylenediaminetetraacetic acid (EDTA). Ethylenediaminetetraacetic acid was used as an anticoagulant to prevent clotting. The samples we immediately transported to the laboratory. Testing was performed with the use of an automated flow cytometry haematology analyser (Roche Diagnostics ${ }^{\circledR}$ ).

\section{T-TAS findings}

Evaluation of whole blood thrombogenicity (assessment of the formation of a thrombus under flow conditions) was assessed immediately after taking the blood samples using T-TAS ${ }^{\circledR}$ at a shear rate of 240 s-1 (Total Thrombus Analysis System, Fujimori Kogyo, Zacros, Japan, AR-chip) equipped with AR microchip and thrombogenic surfaces (collagen with thromboplastin).

For each test, the patient blood samples were analysed for thrombus formation area under a flow pressure curve for 30 min (AUC30 indicates the area under the flow pressure curve for the $30 \mathrm{~min}$. ), time of blood clot formation initiation (T10) and OT or T80 (an occlusion time - time of complete thrombus formation inside the AR-chip). T80-T10 — time of growth of the thrombus on AR chip (duration between the time of initiation of formation of the blood clot, and occlusion time). T10 is defined as the time of the onset of the formation of a thrombus. It resembles the duration of the flow pressure from baseline values to the ten $\mathrm{kPa}$ pressure values. It is related with the partial occlusion of the microcapillary. T80 (OT) represents the time of complete occlusion of the microcapillary, occurring with a pressure of $80 \mathrm{kPa}$. T80-T10 is the interval between T10 and OT or T80 and represents the rate of growth of a thrombus. AUC30 remains an area under the $80 \mathrm{kPa}$ flow pressure curve for $30 \mathrm{~min}$ after the start of the assay in the AR chip.

\section{Statistical analysis}

Statistical analysis was performed using PQ STAT® statistical software. For normality, we checked the data using the Shapiro-Wilk test, and then appropriate parametric or nonparametric tests were used to assess the differences between the parameters across the two 
groups. For parametric, independent samples, the t-Test for independent values and the nonparametric Mann-Whitney U test were used. We used Multivariate logistic regression to obtain the OR values. Due to the limited numbers of participants in the analysed groups, in multivariate logistic regression, the use of only one confounder (BMI) was plausible. The values of the variables are presented as mean \pm one standard deviation or median (interquartile range) appropriate to the use of nonparametric and parametric, respectively. A pvalue of $<0.05$ was considered statistically significant.

The study complies with the principles of the Declaration of Helsinki. The Ethics Committee approved the study protocol of the Poznan University of Medical Sciences (approval number 300/17, 2/3/2017).

We took informed written consent from all patients.

\section{RESULTS}

In this pilot study, we aimed to compare the whole blood thrombus formation process under flow conditions in endometriosis and healthy individuals. Beyond this, we performed conventional standard laboratory investigations in all groups.

\section{Total thrombus analysis system results}

The analysis carried out with the use of T-TAS showed that in women with endometriosis, the initiation of thrombus formation, and the total clot formation time inside the AR-chip occurred significantly faster compared to the control group. The T10 and OT values were significantly shorter between groups ( $\mathrm{p}=0.008$; $\mathrm{p}=0.003$, respectively).

The area under the flow pressure curve for 30 min (AUC) values was significantly higher in the endometriosis group compared to the controls $(p=0.009)$.

Due to the limited number of participants in the analysed groups, in multivariate logistic regression, the use of only one confounder (BMI) was plausible. After analysis adjusted for BMI, a link between an increase by 100 in the AUC-30 values, and a 1.56-fold increased risk of endometriosis was demonstrated, adjusted OR $=1.56[\mathrm{p}=0.01 ; 95 \%$ confidence interval (CI): 1.10-2.18].

\section{Standard laboratory investigations}

We observed differences in the complete blood count (CBC) values in the study and control groups. In the endometriosis group, lower erythrocytes [red blood cells (RBC)], haemoglobin (HGB) concentrations, and the haematocrit value were observed (respectively: p 
$=0.02, \mathrm{p}=0.0008 ; \mathrm{p}=0.03)$. The occurrence of those differences is caused mainly by the heavy menstrual bleeding reported by patients from the study group.

Inflammation status analysis revealed substantially higher leucocytes [white blood cells $(\mathrm{WBC})](\mathrm{p}=0.002)$ as well as neutrophil $(\mathrm{p}=0.00002)$ and basophils $(\mathrm{p}=0.0008)$ concentrations in the study group. Additionally, we observed a statistically lower eosinophils concentration in the endometriosis group $(\mathrm{p}=0.003)$.

The neutrophil-to-lymphocyte ratio (NLR), which is as a marker of subclinical inflammation, was almost threefold higher in the endometriosis group compared to the healthy controls. This difference across those two groups was statistically significant $(\mathrm{p}=$ 0.0001).

Agranulocytes: the lymphocyte and monocyte concentrations did not differ across the groups.

Additionally, there were no differences observed in the PLR (Platelet-to-lymphocyte ratio) between the groups in our study. PLR assessment is a novel index reflecting a systemic inflammatory burden that combines the prognostic values of an individual's platelet and lymphocyte counts.

Our study group comprised of 23 surgically confirmed endometriosis patients. Revised American Society for Reproductive Medicine classification (rASRM) of endometriosis to classify lesions during surgical procedures has been used. With I and II stage of endometriosis were diagnosed 5 and with III and IV 18 patients, respectively. We used only rASRM. Due to non-parametric data median with $1^{\text {st }}$ (lower) and $3^{\text {rd }}$ (upper) quartile were reported in Table 1 . (4; 3-4).

Values and appropriate p-values are shown in Table 1.

\section{DISCUSSION}

In the present study, we investigated the prothrombotic influence of endometriosis using a novel automated microchip flow chamber system for the quantitative analysis of thrombus formation: Total Thrombus-formation Analysis System (T-TAS $\left.{ }^{\circledR}\right)$. To date, there has been no research study assessing the influence of endometriosis on the process of thrombus formation using this system, and no such data has been obtained in women who have endometriosis yet.

T-TAS enables the assessment of the whole blood thrombogenicity [29]. It was designed to evaluate the prophylaxis of various antiplatelet drugs against thrombosis, based on the phenomenon that platelets remain crucial to initiating thrombus formation. This system 
further allows an evaluation of the in vitro thrombus formation process under flow conditions. It is also useful in assessing the clot formation process in haemostasis alterations such as factor VIII deficiency (mouse model) [30]. Previous research on a T-TAS showed that the system might be useful in assessing the effects of antithrombotic agents [29, 31]. Before this study, the impact of endometriosis on haemostasis using T-TAS® had not been analysed.

Increased whole-blood thrombogenicity has been demonstrated in the Endometriosis group.

Augmented blood clotting in those patients is not recognisable using a standard test such as mean platelet value (MPV). Moreover, the platelet count values were not different in the study and control groups. Therefore, our data strongly suggest that T-TAS might be a sensitive diagnostic tool to detect a prothrombotic state occurred in women who have endometriosis, however, our findings need further elucidation in research on significantly more extensive data sets to be confirmed.

There is a considerable body of evidence that endometriosis predisposes to alter the coagulation status in women [32]. Thus, our findings remain consistent with the literature. We support the thesis of a shift towards thrombosis in the haemostasis milieu in that group of patients. Ding et al., showed a higher platelet aggregation rate, fibrinogen concentration, platelet activation rate, elevated plasma D-dimer, plasma soluble P-selectin, prothrombin fragment $1+2(F 1+2)$, fibrin degradation products and shortened thrombin time [33]. In a study conducted by Qinjiao et al., APTT and TT were shortened, and elevated fibrinogen levels were documented. The authors of this study suggested the possibility of a therapeutic role of anticoagulant agents and their potential future use in the treatment of endometriosis [21]. There are numerous studies with a suggestive role in the activation of the coagulation cascade in endometriosis. The potential role of a tissue factor, in the etiopathogenesis of the disease, has been demonstrated as an angiogenic parameter [34].

In this study, we documented that using the T-TAS system. We can detect a significant difference in the time of initiation, elongation and the total time of thrombus formation in healthy vs endometriosis subjects. Moreover, T-TAS not only detects differences in wholeblood thrombogenicity mediated by platelet hyperreactivity but is also sensitive to alterations occurring in the fibrinolytic system. Our results support the thesis that endometriosis is associated with hyperfibrinolysis, and thus they remain consistent with prior research studies on this topic. The fibrinolytic system plays a significant role in maintaining homeostasis. It affects not only the haemostatic balance but also tumour invasion, tissue remodelling, reproduction and angiogenesis [35]. PAI-1 (plasminogen activator inhibitor-1) is a significant 
inhibitor of the fibrinolytic system [36]. A growing body of evidence indicates that increased levels of PAI-1 mediate tumour metastasis, diabetes, cardiovascular and reproductive diseases [37][38][39][40]. Bedaiwy et al., showed that endometriosis is associated with hyperfibrinolysis due to the more frequent occurrence of the 4G allele of the PAI-1 gene in surgically confirmed endometriosis patients [15]. There is, however, a dispute in the link between PAI-1 polymorphic variants and susceptibility to endometriosis [41][42]. Hypofibrinolysis promotes a fibrin matrix, thus supporting the initiation of endometrial lesions in the peritoneal cavity caused by retrograde menstruation [15].

Moreover, in peritoneal fluid from patients with endometriosis, increased PAI-1 antigen links with an increased risk of peritoneal adhesions [42]. PAI-1 regulates tumour cell migration and the mechanism of endometriotic cell invasion [43, 44]. This notion could be potentially a piece of the explanation of why some women develop the endometriosis phenotype while others do not [15]. PAI-1 4G/5G links with endometriosis-associated infertility [45]. PAI-1 antigenic levels were higher in ovarian endometriomas than in a normal eutopic endometrium [46]. In in vitro studies, endometrial stromal tissues sampled from women with endometriosis released higher PAI-1 antigen levels than tissues derived from a healthy control group. The higher release of PAI-1 might be an essential phenomenon in the invasive growth process which occurs in endometriotic lesion formation [43].

The chronic inflammatory process related to the increase of cytokines and chemokines occurring in patients with endometriosis has been well demonstrated in previous research [47].

In our study, we showed a higher NLR (neutrophil-to-lymphocyte ratio) in women with endometriosis. Thus, our results remain consistent with prior research. Tokmak et al. [48], showed higher NLR values in endometriosis patients and stated that the combination of Ca-125 levels and NLR values improves diagnostic accuracy in detecting endometriomas. Cho. et al. [49], evidenced that NLR levels can discriminate endometriosis patients from controls with $83.9 \%$ specificity when combined with other markers such as Ca-125. Our study fails to indicate MPV as a marker of platelet hyperreactivity in women with endometriosis.

Our study group comprised of patients, who underwent surgery due to endometriosis. We have been struggling with defining the reasons for differences in CBC values across the analysed groups, therefore we have stated a hypothesis that those may be related with menstrual disorders, which are more plausible in women suffered from a disease. In authors’ opinion more studies are required to assess eventually plausible linkage between CBC and whole blood thrombogenicity measured by T-TTAS values alterations in women affected by 
endometriosis. Using this limited data, it is very difficult to answer the question whether CBC alteration have affected T-TAS values unequivocally; what may be considered as a limitation of our study.

Summarising, our findings strongly support the thesis that endometriosis triggers a shift toward a prothrombotic and antifibrinolytic state, and this phenomenon can be detectable using a novel microchip flow-chamber system for quantitative assessment of the platelet thrombus formation process under flow conditions in vitro. The results obtained by this system have shown that each increase in AUC (T-TAS $\left.{ }^{\circledR}\right)$ values by 100 increases the risk of developing endometriosis by 1.56 -fold.

Due to the limited data and limited sample size of our study, the role of T-TAS alterations in endometriosis patients needs to further elucidation with much larger data sets in order to confirm our essential findings.

\section{CONCLUSIONS}

The potential thrombotic and antifibrinolytic influence of endometriosis on haemostasis, as well as increased cardiovascular morbidity in women suffering from a disease, have recently been reported in the literature.

T-TAS ${ }^{\circledR}$ (total thrombus formation analysis) is a useful diagnostic tool allowing the assessment of whole blood thrombogenicity. In our study, the (T-TAS ${ }^{\circledR}$ ) results substantially differed in the endometriosis and healthy control groups. Blood clot formation initiation time and occlusion time were significantly shortened in endometriosis, which may indicate the prothrombotic state of this disease. The alteration of (T-TAS ${ }^{2}$ ), the NLR (neutrophil-tolymphocyte ratio) and the granulocytes concentration values in endometriosis support the thesis of a shift of the equilibrium towards thrombosis occurring in patients suffering from the disease, as well as the plausible linkage of this phenomenon with the chronic inflammatory status also found in this group of patients.

\section{Conflict of interest}

The authors declare that there is no conflict of interest regarding the publication of this article.

\section{REFERENCES}

1. Szczepańska M, Wirstlein P, Skrzypczak J, et al. Expression of HOXA11 in the midluteal endometrium from women with endometriosis-associated infertility. Reprod 
Biol Endocrinol. 2012; 10: 1, doi: 10.1186/1477-7827-10-1, indexed in Pubmed: $\underline{22233680 .}$.

2. Cramer DW, Missmer SA. The epidemiology of endometriosis. Ann N Y Acad Sci. 2002; 955: 11-22; discussion 34, doi: 10.1111/j.1749-6632.2002.tb02761.x, indexed in Pubmed: $\underline{11949940 .}$.

3. Szczepańska M, Wirstlein P, Zawadzka M, et al. Alternation of ten-eleven translocation 1, 2, and 3 expression in eutopic endometrium of women with endometriosis-associated infertility. Gynecol Endocrinol. 2018; 34(12): 1084-1090, doi: 10.1080/09513590.2018.1490403, indexed in Pubmed: $\underline{30130982 .}$

4. Andrisani A, Donà G, Brunati AM, et al. Increased oxidation-related glutathionylation and carbonic anhydrase activity in endometriosis. Reprod Biomed Online. 2014; 28(6): 773-779, doi: 10.1016/j.rbmo.2014.01.016, indexed in Pubmed: 24746440.

5. Agic A, Xu H, Finas D, et al. Is endometriosis associated with systemic subclinical inflammation? Gynecol Obstet Invest. 2006; 62(3): 139-147, doi: 10.1159/000093121, indexed in Pubmed: 16679772.

6. Kvaskoff M, Mu F, Terry KL, et al. Endometriosis: a high-risk population for major chronic diseases? Hum Reprod Update. 2015; 21(4): 500-516, doi: 10.1093/humupd/dmv013, indexed in Pubmed: 25765863.

7. Teng SW, Horng HC, Ho CH, et al. Taiwan Association of Gynecology Systematic Review Group. Women with endometriosis have higher comorbidities: Analysis of domestic data in Taiwan. J Chin Med Assoc. 2016; 79(11): 577-582, doi: 10.1016/j.jcma.2016.04.006, indexed in Pubmed: 27553580.

8. Gemmill JA, Stratton P, Cleary SD, et al. Cancers, infections, and endocrine diseases in women with endometriosis. Fertil Steril. 2010; 94(5): 1627-1631, doi: 10.1016/j.fertnstert.2009.07.1698, indexed in Pubmed: 19945097.

9. $\mathrm{Mu}$ F, Rich-Edwards J, Rimm EB, et al. Endometriosis and Risk of Coronary Heart Disease. Circ Cardiovasc Qual Outcomes. 2016; 9(3): 257-264, doi: 10.1161/CIRCOUTCOMES.115.002224, indexed in Pubmed: 27025928.

10. Alhurani RE, Chahal CA, Ahmed AT, et al. Sex hormone therapy and progression of cardiovascular disease in menopausal women. Clin Sci (Lond). 2016; 130(13): 10651074, doi: 10.1042/CS20160042, indexed in Pubmed: 27215679.

11. Lobo RA. Surgical menopause and cardiovascular risks. Menopause. 2007; 14(3 Pt 2): 562-566, doi: 10.1097/gme.0b013e318038d333, indexed in Pubmed: 17476145. 
12. Koumantakis E, Matalliotakis I, Neonaki M, et al. Soluble serum interleukin-2 receptor, interleukin-6 and interleukin-1a in patients with endometriosis and in controls. Arch Gynecol Obstet. 1994; 255(3): 107-112, doi: 10.1007/BF02390936, indexed in Pubmed: 7979562.

13. Pizzo A, Salmeri FM, Ardita FV, et al. Behaviour of cytokine levels in serum and peritoneal fluid of women with endometriosis. Gynecol Obstet Invest. 2002; 54(2): 82-87, doi: 10.1159/000067717, indexed in Pubmed: 12566749.

14. Agic A, Xu H, Altgassen C, et al. Relative expression of 1,25-dihydroxyvitamin D3 receptor, vitamin D 1 alpha-hydroxylase, vitamin D 24-hydroxylase, and vitamin D 25-hydroxylase in endometriosis and gynecologic cancers. Reprod Sci. 2007; 14(5): 486-497, doi: 10.1177/1933719107304565, indexed in Pubmed: 17913968.

15. Bedaiwy MA, Falcone T, Mascha EJ, et al. Genetic polymorphism in the fibrinolytic system and endometriosis. Obstet Gynecol. 2006; 108(1): 162-168, doi: 10.1097/01.AOG.0000220517.53892.0a, indexed in Pubmed: 16816071.

16. Szczepańska M, Mostowska A, Wirstlein P, et al. Polymorphic variants in vitamin D signaling pathway genes and the risk of endometriosis-associated infertility. Mol Med Rep. 2015; 12(5): 7109-7115, doi: 10.3892/mmr.2015.4309, indexed in Pubmed: 26398313.

17. Dionigi R. Effects of surgical trauma of laparoscopic vs. open cholecystectomy. Hepatogastroenterology. 1994; 41(5): 471-476.

18. Jilma B, Blann A, Pernerstorfer T, et al. Regulation of adhesion molecules during human endotoxemia. No acute effects of aspirin. Am J Respir Crit Care Med. 1999; 159(3): 857-863, doi: 10.1164/ajrccm.159.3.9805087, indexed in Pubmed: 10051263.

19. Ding S, Lin Q, Zhu T, et al. Is there a correlation between inflammatory markers and coagulation parameters in women with advanced ovarian endometriosis? BMC Womens Health. 2019; 19(1): 169, doi: 10.1186/s12905-019-0860-9, indexed in Pubmed: $\underline{31888633}$.

20. Velasco I, Acién P, Campos A, et al. Interleukin-6 and other soluble factors in peritoneal fluid and endometriomas and their relation to pain and aromatase expression. J Reprod Immunol. 2010; 84(2): 199-205, doi: 10.1016/j.jri.2009.11.004, indexed in Pubmed: 20074813.

21. Wu Q, Ding D, Liu X, et al. Evidence for a Hypercoagulable State in Women With Ovarian Endometriomas. Reprod Sci. 2015; 22(9): 1107-1114, doi: 10.1177/1933719115572478, indexed in Pubmed: 25701841. 
22. Lipinski S, Bremer L, Lammers T, et al. Coagulation and inflammation. Molecular insights and diagnostic implications. Hamostaseologie. 2011; 31(2): 94-102, 104, doi: 10.5482/ha-1134, indexed in Pubmed: 21152678.

23. Petäjä J. Inflammation and coagulation. An overview. Thrombosis Research. 2011; 127: S34-S37, doi: 10.1016/s0049-3848(10)70153-5.

24. Hirota Y, Osuga Y, Hirata T, et al. Possible involvement of thrombin/proteaseactivated receptor 1 system in the pathogenesis of endometriosis. J Clin Endocrinol Metab. 2005; 90(6): 3673-3679, doi: 10.1210/jc.2004-0493, indexed in Pubmed: 15755869 .

25. Hirota Y, Osuga Y, Hirata T, et al. Activation of protease-activated receptor 2 stimulates proliferation and interleukin (IL)-6 and IL-8 secretion of endometriotic stromal cells. Hum Reprod. 2005; 20(12): 3547-3553, doi: 10.1093/humrep/dei255, indexed in Pubmed: $\underline{16096323}$.

26. Ding D, Liu X, Duan J, et al. Platelets are an unindicted culprit in the development of endometriosis: clinical and experimental evidence. Hum Reprod. 2015; 30(4): $812-$ 832, doi: 10.1093/humrep/dev025, indexed in Pubmed: 25740881.

27. Liu J, Liu X, Li Y, et al. The association of neutrophil to lymphocyte ratio, mean platelet volume, and platelet distribution width with diabetic retinopathy and nephropathy: a meta-analysis. Biosci Rep. 2018; 38(3), doi: 10.1042/BSR20180172, indexed in Pubmed: 29581246.

28. Lipets EN, Ataullakhanov FI. Global assays of hemostasis in the diagnostics of hypercoagulation and evaluation of thrombosis risk. Thromb J. 2015; 13(1): 4, doi: 10.1186/s12959-015-0038-0, indexed in Pubmed: 25635172.

29. Hosokawa K, Ohnishi T, Kondo T, et al. A novel automated microchip flow-chamber system to quantitatively evaluate thrombus formation and antithrombotic agents under blood flow conditions. J Thromb Haemost. 2011; 9(10): 2029-2037, doi: 10.1111/j.1538-7836.2011.04464.x, indexed in Pubmed: 21827607.

30. Ogawa S, Szlam F, Dunn AL, et al. Evaluation of a novel flow chamber system to assess clot formation in factor VIII-deficient mouse and anti-factor IXa-treated human blood. Haemophilia. 2012; 18(6): 926-932, doi: 10.1111/j.1365-2516.2012.02867.x, indexed in Pubmed: 22642581.

31. Hosokawa K, Ohnishi T, Fukasawa M, et al. A microchip flow-chamber system for quantitative assessment of the platelet thrombus formation process. Microvasc Res. 
2012; 83(2): 154-161, doi: 10.1016/j.mvr.2011.11.007, indexed in Pubmed: $\underline{22166857 .}$.

32. Viganò P, Ottolina J, Sarais V, et al. Coagulation Status in Women With Endometriosis. Reprod Sci. 2018; 25(4): 559-565, doi: 10.1177/1933719117718273, indexed in Pubmed: 28681683.

33. Ding D, Liu X, Guo SW. Further Evidence for Hypercoagulability in Women With Ovarian Endometriomas. Reprod Sci. 2018; 25(11): 1540-1548, doi: 10.1177/1933719118799195, indexed in Pubmed: $\underline{30244655}$.

34. Krikun G, Schatz F, Taylor H, et al. Endometriosis and tissue factor. Ann N Y Acad Sci. 2008; 1127: 101-105, doi: 10.1196/annals.1434.008, indexed in Pubmed: 18443336.

35. Zorio E, Gilabert-Estellés J, España F, et al. Fibrinolysis: the key to new pathogenetic mechanisms. Curr Med Chem. 2008; 15(9): 923-929, doi: 10.2174/092986708783955455, indexed in Pubmed: 18473800.

36. Hellgren M. Hemostasis during normal pregnancy and puerperium. Semin Thromb Hemost. 2003; 29(2): 125-130, doi: 10.1055/s-2003-38897, indexed in Pubmed: 12709915.

37. Ye Y, Vattai A, Zhang Xi, et al. Role of Plasminogen Activator Inhibitor Type 1 in Pathologies of Female Reproductive Diseases. Int J Mol Sci. 2017; 18(8), doi: 10.3390/ijms18081651, indexed in Pubmed: 28758928.

38. Cesarman-Maus G, Hajjar KA. Molecular mechanisms of fibrinolysis. Br J Haematol. 2005; 129(3): 307-321, doi: 10.1111/j.1365-2141.2005.05444.x, indexed in Pubmed: 15842654.

39. Duffy MJ, McGowan PM, Harbeck N, et al. uPA and PAI-1 as biomarkers in breast cancer: validated for clinical use in level-of-evidence-1 studies. Breast Cancer Res. 2014; 16(4): 428, doi: 10.1186/s13058-014-0428-4, indexed in Pubmed: 25677449.

40. Lyon CJ, Hsueh WA. Effect of plasminogen activator inhibitor-1 in diabetes mellitus and cardiovascular disease. Am J Med. 2003; 115 Suppl 8A: 62S-68S, doi: 10.1016/j.amjmed.2003.08.014, indexed in Pubmed: 14678868.

41. Gentilini D, Vigano P, Castaldi D, et al. Plasminogen activator inhibitor-1 4G/5G polymorphism and susceptibility to endometriosis in the Italian population. Eur J Obstet Gynecol Reprod Biol. 2009; 146(2): 219-221, doi: 10.1016/j.ejogrb.2009.06.008, indexed in Pubmed: 19608328. 
42. Ramón LA, Gilabert-Estellés J, Cosín R, et al. Plasminogen activator inhibitor-1 (PAI1) 4G/5G polymorphism and endometriosis. Influence of PAI-1 polymorphism on PAI-1 antigen and mRNA expression. Thromb Res. 2008; 122(6): 854-860, doi: 10.1016/j.thromres.2008.02.010, indexed in Pubmed: 18423526.

43. Bruse C, Guan Y, Carlberg M, et al. Basal release of urokinase plasminogen activator, plasminogen activator inhibitor-1, and soluble plasminogen activator receptor from separated and cultured endometriotic and endometrial stromal and epithelial cells. Fertil Steril. 2005; 83 Suppl 1: 1155-1160, doi: 10.1016/j.fertnstert.2004.09.033, indexed in Pubmed: 15831288.

44. Greene AD, Lang SA, Kendziorski JA, et al. Endometriosis: where are we and where are we going? Reproduction. 2016; 152(3): R63-R78, doi: 10.1530/REP-16-0052, indexed in Pubmed: 27165051.

45. Gonçalves-Filho RP, Brandes A, Christofolini DM, et al. Plasminogen activator inhibitor-1 4G/5G polymorphism in infertile women with and without endometriosis. Acta Obstet Gynecol Scand. 2011; 90(5): 473-477, doi: 10.1111/j.16000412.2011.01086.x, indexed in Pubmed: 21306344.

46. Gilabert-Estellés J, Estellés A, Gilabert J, et al. Expression of several components of the plasminogen activator and matrix metalloproteinase systems in endometriosis. Hum Reprod. 2003; 18(7): 1516-1522, doi: 10.1093/humrep/deg300, indexed in Pubmed: 12832381.

47. Burney RO, Giudice LC. Pathogenesis and pathophysiology of endometriosis. Fertil Steril. 2012; 98(3): 511-519, doi: 10.1016/j.fertnstert.2012.06.029, indexed in Pubmed: 22819144.

48. Tokmak A, Yildirim G, Öztaş E, et al. Use of Neutrophil-to-Lymphocyte Ratio Combined With CA-125 to Distinguish Endometriomas From Other Benign Ovarian Cysts. Reprod Sci. 2016; 23(6): 795-802, doi: 10.1177/1933719115620494, indexed in Pubmed: 26692541.

49. Cho S, Cho H, Nam A, et al. Neutrophil-to-lymphocyte ratio as an adjunct to CA-125 for the diagnosis of endometriosis. Fertil Steril. 2008; 90(6): 2073-2079, doi: 10.1016/j.fertnstert.2008.03.061, indexed in Pubmed: 18555226.

Table 1. Characteristics of the studied groups 


\begin{tabular}{|l|l|l|l|l|l|l|c|}
\hline VARIABLE & CONTROL* & $\begin{array}{l}\text { SD/ LOWER } \\
\text { QUARTILE* }\end{array}$ & $\begin{array}{l}\text { UPPER } \\
\text { QUARTILE* }\end{array}$ & $\begin{array}{l}\text { ENDOMETRIOSIS } \\
*\end{array}$ & $\begin{array}{l}\text { SD/ LOWER } \\
\text { QUARTILE* }\end{array}$ & $\begin{array}{l}\text { UPPER } \\
\text { QUARTILE* }\end{array}$ & $\mathrm{p}^{* *}$ \\
\hline
\end{tabular}




\begin{tabular}{|c|c|c|c|c|c|c|c|}
\hline & $\mathrm{n}=10$ & & & $\mathrm{n}=23$ & & & \\
\hline BMI $\left[\mathrm{kg} / \mathrm{m}^{2}\right]$ & 21.55 & 20.56 & 23.83 & 22.00 & 19.76 & 23.55 & 0.8 \\
\hline $\begin{array}{l}\text { ENDOMETRIOSIS } \\
\text { STAGE [rASRM] }\end{array}$ & N/A & & & $\begin{array}{c}4 \\
(\mathrm{I}=2 ; \mathrm{II}=3 ; \mathrm{III}=5 ; \\
\mathrm{IV}=13)\end{array}$ & 3 & 4 & - \\
\hline \multicolumn{8}{|c|}{ Comparative laboratory investigations } \\
\hline $\begin{array}{l}\text { ERYTHROCYTES } \\
\text { (RBC) [G/L] }\end{array}$ & 4.45 & 4.33 & 4.65 & 4.07 & 3.99 & 4.47 & 0.02 \\
\hline $\begin{array}{l}\text { RDW (RED CELL } \\
\text { DISTRIBUTION } \\
\text { WIDTH) [\%] }\end{array}$ & 12.95 & 12.38 & 13.35 & 13.10 & 12.25 & 13.45 & 0.16 \\
\hline MCV [fL] & 85.26 & 3.08 & & 85.11 & 5.34 & & 0.96 \\
\hline $\mathrm{MCH}[\mathrm{fmol}]$ & 1.92 & 1.78 & 1.94 & 1.81 & 1.70 & 1.86 & 0.07 \\
\hline $\mathrm{MCHC}[\mathrm{mmol} / \mathrm{L}]$ & 21.76 & 0.80 & & 20.72 & 0.76 & & 0.001 \\
\hline $\mathrm{HGB}[\mathrm{mmol} / \mathrm{L}]$ & 8.32 & 0.61 & & 7.44 & 0.63 & & 0.0008 \\
\hline $\mathrm{HCT}[\mathrm{L} / \mathrm{L}]$ & 0.38 & 0.02 & & 0.36 & 0.03 & & 0.03 \\
\hline $\begin{array}{l}\text { LEUKOCYTES } \\
(\mathrm{WBC})[\mathrm{G} / \mathrm{L}]\end{array}$ & 6.18 & 4.82 & 6.55 & 9.88 & 6.22 & 12.02 & 0.002 \\
\hline $\begin{array}{l}\text { NEUTROPHILES } \\
{[\mathrm{G} / \mathrm{L}]}\end{array}$ & 3.23 & 1.03 & & 7.91 & 4.06 & & 0.00002 \\
\hline $\begin{array}{l}\text { EOZYNOPHYLES } \\
{[\mathrm{G} / \mathrm{L}]}\end{array}$ & 0.10 & 0.09 & 0.16 & 0.04 & 0.02 & 0.085 & 0.003 \\
\hline $\begin{array}{l}\text { BASOPHYLES } \\
{[\mathrm{G} / \mathrm{L}]}\end{array}$ & 0.02 & 0.02 & 0.02 & 0.04 & 0.03 & 0.045 & 0.0008 \\
\hline $\begin{array}{l}\text { LYMPHOCYTES } \\
{[\mathrm{G} / \mathrm{L}]}\end{array}$ & 1.88 & 0.68 & & 1.66 & 0.55 & & 0.33 \\
\hline $\begin{array}{l}\text { MONOCYTES } \\
{[\mathrm{G} / \mathrm{L}]}\end{array}$ & 0.49 & 0.44 & 0.53 & 0.67 & 0.42 & 0.82 & 0.16 \\
\hline PLATELETS [G/L] & 270.50 & 206.00 & 279.00 & 255.00 & 219.50 & 291 & 0.52 \\
\hline MPV [fl] & 11.12 & 0.67 & & 10.56 & 0.67 & & 0.03 \\
\hline $\begin{array}{l}\text { Platelet-to- } \\
\text { lymphocyte ratio } \\
\text { (PLR) }\end{array}$ & 133.27 & 119.51 & 181.01 & 164.36 & 125.07 & 203.99 & 0.36 \\
\hline $\begin{array}{l}\text { Neutrophil-to- } \\
\text { lymphocyte ratio } \\
\text { (NLR) }\end{array}$ & 1.66 & 1.41 & 2.11 & 4.79 & 3.11 & 6.34 & 0.0001 \\
\hline $\begin{array}{l}\text { ENDOMETRIOSIS } \\
\text { STAGE }\end{array}$ & N/A & & & 4 & 3 & 4 & - \\
\hline \multicolumn{8}{|c|}{ Comparative T-TAS findings } \\
\hline $\mathrm{T} 10[\mathrm{~s}]$ & 639.40 & 284.07 & & 325.70 & 165.45 & & 0.008 \\
\hline AUC30 & 1468.65 & 398.35 & & 1893.40 & 233.68 & & 0.009 \\
\hline OT [s] & 800.50 & 599.50 & 926.50 & 385.00 & 328.50 & 511 & 0.003 \\
\hline
\end{tabular}

${ }^{*}$ Mean with SD (standard deprivation) are presented for parametric data. Median with $1^{\text {st }}$ (lower) and $3^{\text {rd }}$ (upper) quartile are reported for non-parametric data; **For parametric: 
independent-samples t-Test and for nonparametric U Mann-Whitney test were used; Note: Statistically significant p values are indicated in bold; BMI — body mass index; N/A — not applicable; RBC — red blood cells; HGB — haemoglobin; WBC — white blood cells; MPV — mean platelet volume; T10 — time of blood clot formation initiation; AUC30 — area under the curve; OT — occlusion time 